\title{
OSNR Prediction Based on Federal Learning in Multi-Domain Optical Networks
}

\author{
Junhua HUANG ${ }^{\mathrm{a}}$, Bohan ZHU ${ }^{\mathrm{b}, 1}$, Hongxi ZHOU ${ }^{\mathrm{a}}$, Qiwei ZHENG ${ }^{\mathrm{a}}$, Zhuo CHEN ${ }^{\mathrm{a}}$, \\ Tong LIN ${ }^{\mathrm{a}}$, Yuanyuan ZHAO ${ }^{\mathrm{a}}$, Ziqi DONG ${ }^{\mathrm{a}}$, Xiaofang $\mathrm{GAO}^{\mathrm{a}}$, Yongli ZHAO ${ }^{\mathrm{b}}$ \\ anformation and Communication Branch of State Grid Corporation of China, China \\ ${ }^{\mathrm{b}}$ Beijing University of Posts and Telecommunications, China
}

\begin{abstract}
With the continuous expansion of the scale of optical communication network and the rapid increase of network traffic demand, the management form of multi-domain optical network has widely existed. OSNR is an important indicator to judge the quality of communication. It is very important to predict OSNR more accurately in a low-cost and energy-saving way in multi-domain optical networks. In this paper, a scheme of federal learning in multi-domain optical networks is proposed to improve the accuracy of the OSNR prediction. The main idea is to train hybrid machine learning model in each single domain, then the strategy of federal learning is used for optimization it in multi-domains. The performance of the proposed scheme is verified by simulation experiments. The strategy can alleviate the problems of data silos and model training set caused by multi-domain optical network. According to simulation result, when the amount of data reaches $5 \times 103$, adding this strategy will reduce the mean square error of the prediction model by about $18 \%$. It can improve the performance of machine learning model, the ability of OSNR prediction and the reliability of network operation.
\end{abstract}

Keywords. OSNR prediction, federal learning in multi-domain, optical networks

\section{Introduction}

Optical communication networks have the advantages of large bandwidth and low latency. It is an important foundation of the next generation of communication technology [1]. With the increasing scale of optical networks, a large-scale optical communication network is divided into multiple small-scale optical networks according to various dimensions such as geographical locations, managers and equipment types. Each divided small-scale optical network is called an optical network domain, and each optical network domain is managed by its own domain managers.

OSNR has long been widely used in the study of optical communication networks. The value of OSNR directly reflects the transmission quality of signals [2]. OSNR can be considered as a condition in routing and network optimization algorithms. With the increasing diversity of communication services, data silos and model training sets are new challenges to OSNR prediction. Besides, due to the bottleneck of the controller and

\footnotetext{
${ }^{1}$ Corresponding Author: 1285603132@qq.com
} 
data acquisition mechanism, multi-domain network management constraints and other reasons, the amount of data monitored in their respective management domains is small, the scale of the single domain network will not be too large. Traditional OSNR prediction model cannot exchange data well between domains, and it has high requirements for training samples in a single domain. To sum up, it's more and more difficult for traditional OSNR prediction model to play a role in the multi-domain optical network system.

There are two major directions for OSNR prediction of optical communication equipment. One is based on the prior knowledge scheme of analytical formula. In reference [3], the prediction of OSNR based on power and noise is proposed. The value of OSNR can be calculated by analytical formula through the measured power and noise, which is limited by whether the power and noise can be accurately calculated. In reference [4], it is proposed to monitor OSNR indexes through high-order statistical moments. However, as the evolution process of optical network technology, traditional OSNR monitoring acquisition method does not capture the many important OSNR characteristics. The other direction is based on the posterior knowledge scheme of historical data and machine learning algorithm. Artificial intelligence algorithm is a posteriori knowledge scheme. With the help of historical data, it learns data features through algorithm model and obtains calculation results. In reference [5], a deep learning system based on artificial neural network dynamically predicts the OSNR value of optical communication equipment by monitoring modulation format, channel occupancy rate, power, link length, EDFA noise figure and other parameters. In reference [6], an OSNR calculation method combining cyclic neural network and attention mechanism is proposed. However, it is difficult for these AI models to be directly applied to actual scenarios, because they require many data sets for model training. However, in actual scenarios, there are few effective device performance data, which may fail to meet the requirements of prediction accuracy.

In this paper, an OSNR prediction algorithm based on hybrid machine learning model is proposed. The main idea is to train hybrid machine learning model in each single domain, which combines a priori knowledge with a posteriori knowledge. Then the strategy of federal learning is used for optimization it in multi-domains [7]. Superiority of the OSNR prediction algorithm based on hybrid machine learning model is verified by simulation. Compared with the analytical formula method, the accuracy is higher, and the training complexity is lower than the pure original data-driven machine learning algorithm. Finally, based on the hybrid machine learning model, the strategy of federal learning in multi-domain is introduced, and the OSNR prediction algorithm flow based on federal learning is proposed. The effectiveness of the algorithm is verified by simulation experiments. Compared with acquiring knowledge from a single network domain, the technical scheme of acquiring knowledge from multi-domain and multisource has better performance. Mean square error (MSE) of OSNR prediction model is $18 \%$ smaller when the modal optimized with strategy of federal learning in multi-domain.

\section{Hybrid Machine Learning Model}

Hybrid machine learning model combines a priori knowledge with a posteriori knowledge. By introducing prior knowledge into the machine learning model based on posterior knowledge, the training complexity of the algorithm can be reduced. It should be noted that in this paper, it is only to prove that the hybrid machine learning model 
composed of introducing prior knowledge into the posterior knowledge model has better performance. Different prior knowledge models and a posteriori knowledge models can be selected for different network scenarios to improve performance. In this paper, the OSNR calculation method in reference [3] is used as a prior knowledge, and the calculation is shown in equation (1) and equation (2). The deep neural network model is selected as a posteriori knowledge to learn the -logical relationship of historical data.

$$
\begin{aligned}
& \text { OSNR }=P_{\text {out }}-P_{A S E} \\
& P_{A S E}=-58+N_{F}+G
\end{aligned}
$$

$O S N R$ is the value calculated by using the algorithm based on prior knowledge; $P_{\text {out }}$ is the output power of the optical communication equipment; $P_{A S E}$ is the noise power of the output end of the optical communication equipment; the amplifier noise power $N_{F}$ is calculated from the amplifier noise figure and the amplifier gain $G$.

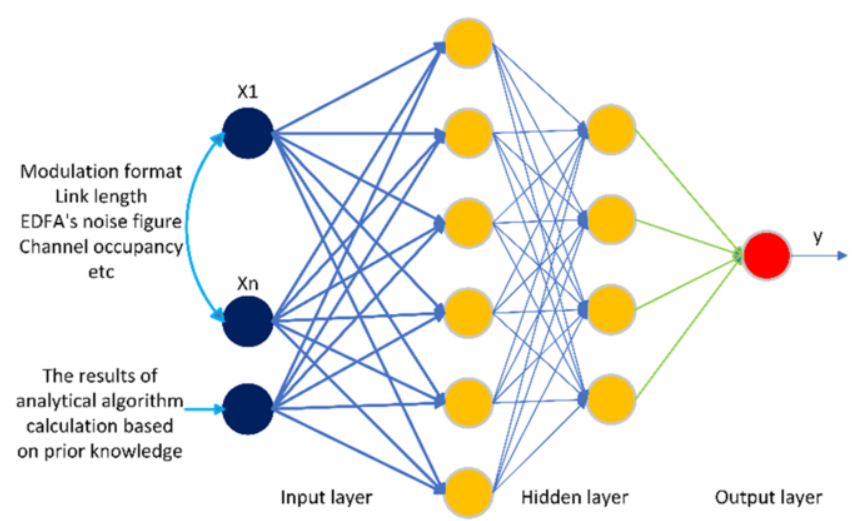

Figure 1. Network structure diagram based on hybrid machine learning model

For the traditional machine learning model, the modulation format, link length, and the noise figure, resource usage and power of the erbium-doped optical fiber amplifier (EDFA) are used as inputs of deep neural networks [8,9], which can be used to extract complex features through multiple nonlinear hidden layers. Then, the nonlinear relationship between input and output is obtained, and the calculated OSNR value is output at the output layer. For the hybrid machine learning model, an additional input parameter is added compared with the traditional machine learning model, which is the result of calculation based on prior knowledge. The outputs are the same. Figure 1 shows the network structure of hybrid machine learning model.

\section{Knowledge Fusion Strategy Scheme}

In this section, a knowledge fusion strategy is proposed for federal learning of multidomain in optical networks (as shown in Figure 2). In essence, the scheme uses the local network domain and other domain models to make federal decisions, so that the domain controller can obtain knowledge from a single source to multiple sources. This scheme 
eases the problem of data silos between multiple domains in optical network, makes the data exchange between domains smoother.

The machine learning model is represented by a series of parameters (the structure of deep neural network, the number of layers, the number of hidden layer nodes and the weight coefficient after network training, etc.). Each trained model consists of specific vector parameters $\theta$ Composed of. Each machine learning model can receive a series of input characteristic data $\mathrm{X}$ and output the value of prediction $\mathrm{Y}$, which can be numerical. This machine learning model and additional metadata $\pi$ ( $\pi$ stores some parameters required for knowledge fusion) are shared among domains as a combination.

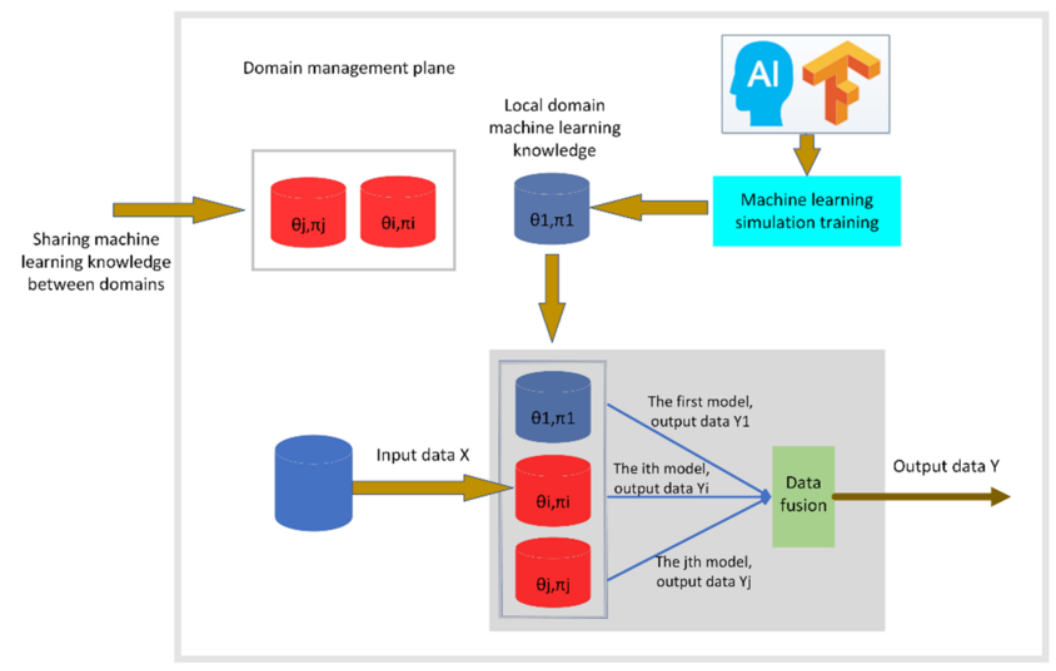

Figure 2. Knowledge fusion strategy for multi-domain optical networks

The specific steps of knowledge fusion are as follows: firstly, the machine learning model trained through the local domain network data in the local domain can be transferred to the local machine learning model. If the model can meet the performance requirements, the network decision can be made, and the knowledge can be shared among alternative domains to other domains. Once the data sample size is insufficient or accuracy of OSNR prediction is too low, you can request high-quality models in other domains for collaborative decision-making; Then, after requesting high-quality models in other domains in the local domain, input the characteristic data into all models to obtain their own model output $\mathrm{Y}_{\mathrm{i}}$. Finally, through the data fusion operation (for the calculation of regression operation equation (3), the result with the highest output frequency can be taken as the final output data for the classification task), the output results $Y_{i}$ of all models are fused to obtain the final output result $Y$.

$$
Y=\frac{Y_{1} f_{1}+Y_{2} f_{2}+\ldots \ldots+Y_{n} f_{n}}{n}
$$

$\mathrm{Y}$ is the final output data, $\mathrm{Y}_{1}, \mathrm{Y}_{2}$ and $\mathrm{Y}_{\mathrm{n}}$ are the output data of each machine learning model, and $\mathrm{n}$ is the number of machine learning models. 


\section{OSNR Prediction Strategy of Federal Learning in Multi-Domain}

This section mainly discusses the process of OSNR prediction algorithm based on federal learning strategy in multi-domain optical networks. It is assumed that the OSNR prediction model in a single domain has been trained, and there will be a trained hybrid machine learning model in each single domain network for OSNR prediction. As shown in the Figure 3, The OSNR prediction technology scheme based on federal learning in multi-domain is mainly divided into three steps:

Step 1 is for the controller to collect historical network data. The collected data types include modulation formats, link lengths, noise coefficients of EDFA, resource occupancy rates, power, and the network characteristic data required by the prior knowledge calculation formula. The results are then calculated by prior knowledge, and the calculated results are combined with other network data into the input feature vector required by the hybrid machine learning model (as shown in Figure 1 input neuron).

Step 2 is the data transmission process, and the input eigenvectors combined in step 1 are input into the trained hybrid machine learning model.

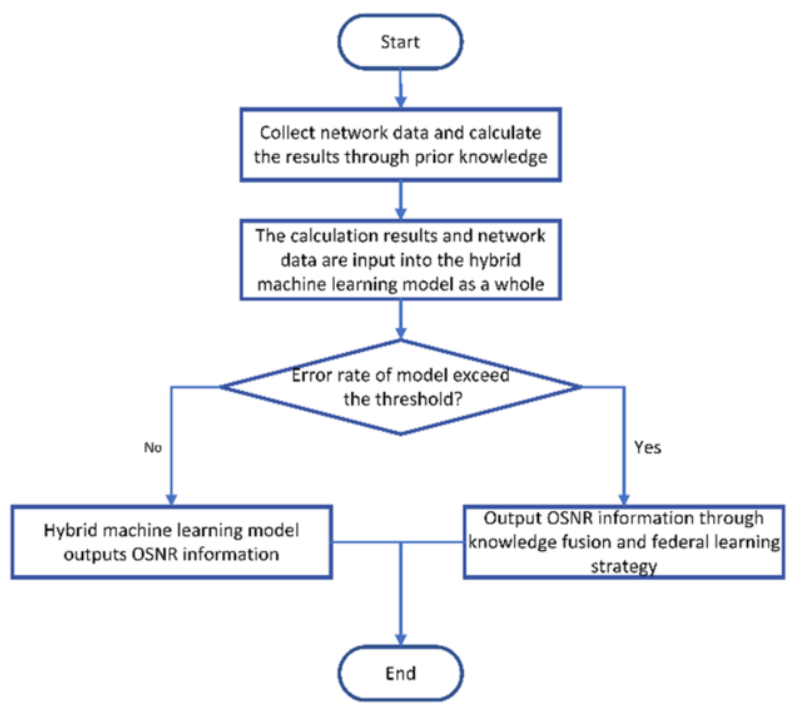

Figure 3. Flow chart of OSNR prediction based on multi-domain in federal learning.

Step 3 is the availability judgment step of hybrid machine learning. The network controller maintains a recent prediction error rate for the original local network domain model. When the prediction error rate reaches the set threshold, the strategy of federal learning in multi-domain will be started. Through knowledge fusion, the strategy of federal learning in multi-domain will replace the output of the original model as optical signal-to-noise ratio information. If the error rate does not reach the set threshold, the multi strategy of federal learning in multi-domain switch will be turned off. The OSNR is predicted directly through the existing single network domain hybrid machine learning model. 
So far, through the above three steps, by collecting the network characteristic data required by the hybrid machine learning model and finally outputting the OSNR information, other network applications can obtain the OSNR information and take corresponding network decisions, such as considering the OSNR constraints in routing calculation. Accurate prediction of OSNR can provide a solid foundation for network traffic engineering and enhance the healthy operation of the network.

\section{Simulation Analysis of OSNR Prediction Based on Federal Learning in Multi- Domain}

To verify the reliability and effectiveness of OSNR prediction algorithm based on hybrid machine learning model, this paper simulates optical communication equipment through Python language and Gnpy optical network planning tool library [10,11]. The system diagram is shown in Figure 4. Different data are generated by changing communication parameters (such as adjusting format, power, amplifier parameters, link length, etc.). The data is expanded by adding white noise to the data, and then the generated data set is divided into two parts, namely, training set and verification set. The hybrid machine learning model is generated through the training set, and the model is verified on the generated verification set.

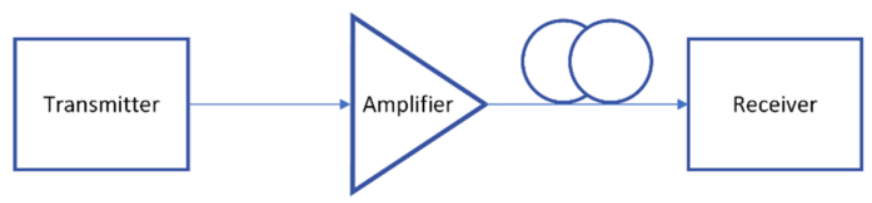

Figure 4. Schematic diagram of the system for generating experimental data.

This paper uses the hybrid machine learning model described in section 2 to predict the OSNR of the generated data. The prior knowledge results are calculated by equations (1) and (2). The posterior knowledge selects the deep neural network model. After a lot of attempts, a group of stable network structure is finally selected.

Firstly, the accuracy of the hybrid machine learning model with full rich data samples and training time at convergence is verified. The MSE predicted by OSNR are $0.295 \mathrm{db}$ (analytical formula model), $0.144 \mathrm{db}$ (machine learning model) and $0.136 \mathrm{db}$ (hybrid machine learning model) respectively. Compared with machine learning model, the error of hybrid machine learning model is reduced by $5 \%$. It is worth noting that in the case of limited data and training time, the hybrid machine learning model has better performance than the machine learning model. In Figure 5(a), we can see the convergence process of the adopted model, in which the hybrid machine learning model has faster speed. More specifically, when the goal of MSE is $0.2 \mathrm{db}$, the time required for hybrid machine learning is 34\% less than that of machine learning model (using the same neural network configuration and the same training parameters). It can be found that the training cycle required by hybrid machine learning model is slightly shorter than that of machine learning model. 

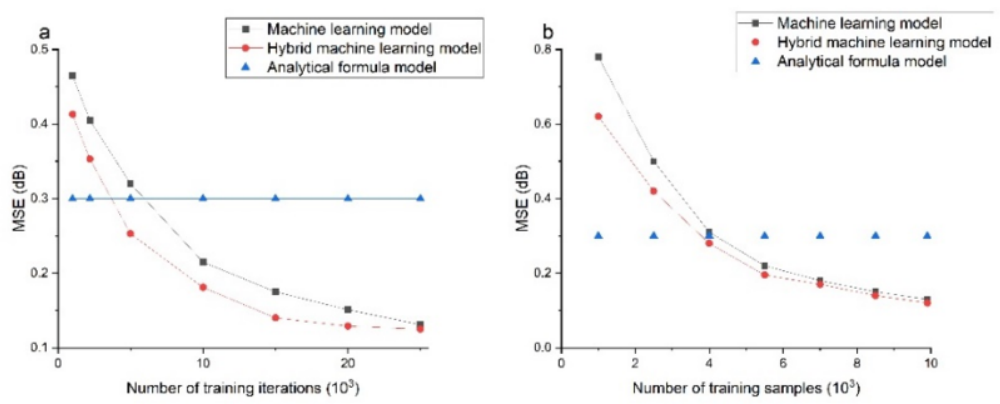

Figure 5. (a) Comparison of training time of different models. (b) Comparison s of training samples in different model.

Figure 5(b) shows the MSE of the three models under different sample numbers. In the case of fewer training samples, the hybrid machine learning model can achieve similar performance to the machine learning model. To finally achieve the result of 0.144 $\mathrm{dB}$ of the machine learning model, the hybrid machine learning model can reduce the training samples by $25 \%$. Moreover, to exceed the prediction accuracy of the analytical formula model, the hybrid machine learning model has about $12 \%$ less training samples than the traditional machine learning model. The experimental results show that the amount of training data required by the hybrid machine learning model is greater than or equal to the machine learning model to achieve the performance like the machine learning model.

To verify the optimization of the strategy of federal learning in multi-domain on the OSNR prediction scene, two data of the same scale are generated according to the data generation mode through the Gnpy library, which is used to simulate other network domain data in the multi-domain network and use the three data to train three hybrid machine learning models. The control experiment is formed by comparing with the model trained with only full single domain data. Figure 6 shows the MSE of the hybrid machine learning model before and after the introduction of strategy of federal learning in multi-domain. It can be seen from the figure that for the OSNR prediction model with federal learning is introduced, the MSE is $18 \%$ smaller when the final model converges, which means that the model optimized with strategy of federal learning in multi-domain

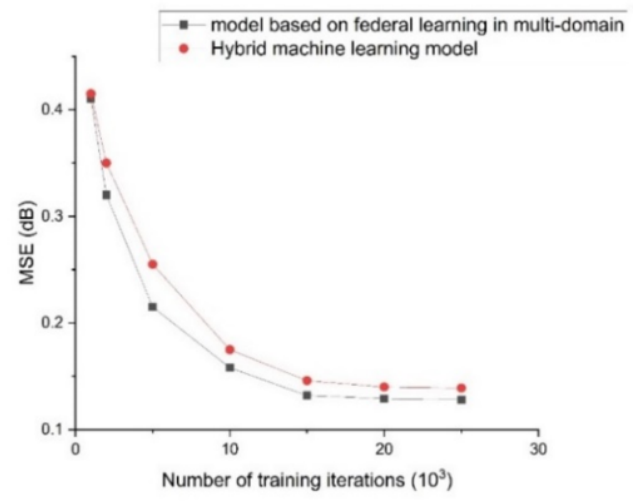

Figure 6. Renderings of fusion strategy of federal learning in multi-domain. 
has better performance and higher prediction accuracy. To sum up, the introduction of this strategy can optimize the model performance of the local network domain and obtain a model with better performance.

\section{Conclusions}

This paper analyzes the problem of OSNR prediction and its related technologies in detail and discusses the scheme evolution of OSNR prediction in single domain network in detail. Starting from the prior knowledge scheme based on analytical formula and the posteriori knowledge scheme based on machine learning. In this paper, a hybrid machine learning model based on prior knowledge and posteriori knowledge is proposed to predict the OSNR, and then the application of strategy of federal learning in multidomain to OSNR prediction is discussed. Then the algorithm flow of OSNR based on multi-domain optical network federal learning is described. Finally, the performance of the proposed strategy scheme is verified by simulation experiments. This strategy scheme can alleviate the problems of data silos and model training set caused by multidomain optical network. The experimental results show that in a certain network domain. The algorithm with strategy of federal learning in multi-domain has better performance than the model algorithm obtained only with local data sets.

\section{Acknowledgments}

This work has been supported in part by National Natural Science Foundation of China (NSFC) (62021005, 61822105).

\section{References}

[1] F5G Whitepaper rev5 [EB/OL]. 2020-07-30. https://portal.etsi.org/ngppapp/Contribution Creation.aspx?primarykeys $=204101$

[2] Z. Wang, A. Yang, P. Guo, L. Feng and P. He, "CNN based OSNR estimation method for long haul optical fiber communication systems," 2018 Asia Communications and Photonics Conference (ACP), 2018, pp. 1-3, doi: 10.1109/ACP.2018.8596136.

[3] Samuel A L. Some Studies in Machine Learning Using the Game of Checkers. II-Recent Progress[J]. 1988.

[4] Hui Yang,Jie Zhang,Yongli Zhao,Jianrui Han,Yi Lin,Young Lee. Cross stratum optimization for software defined multi-domain and multi-layer optical transport networks deploying with data centers[J]. Optical Switching and Networking,2015.

[5] Morii M, Ikeda S, Tominaga N, et al. Machine-learning Selection of Optical Transients in Subaru/Hyper Suprime-Cam Survey[J]. Publications of the Astronomical Society of Japan, 2016, 68(6): 104.

[6] Musumeci F, Rottondi C, Nag A, et al. An Overview on Application of Machine Learning Techniques in Optical Networks[J]. IEEE Communications Surveys \& Tutorials, 2018:1-1.

[7] Lili Zhang, Yuxiang Xie, Luan Xidao and Xin Zhang, "Multi-source heterogeneous data fusion," 2018 International Conference on Artificial Intelligence and Big Data (ICAIBD), Chengdu, 2018, pp. 47-51.

[8] M. J. da Silva et al., "Investigation of Spectral Tilt and OSNR in Cascade of EDFA with Linear Signal Power Pre-emphasis," 2019 SBMO/IEEE MTT-S International Microwave and Optoelectronics Conference (IMOC), 2019, pp. 1-3, doi: 10.1109/IMOC43827.2019.9317623.

[9] Y. Yamamoto, M. Hirano, S. Oda, Y. Aoki, K. Sone and J. C. Rasmussen, "Impact of fiber loss and Aeff on OSNR improvement for hybrid-Raman/EDFA amplified systems," 2015 Opto-Electronics and Communications Conference (OECC), 2015, pp. 1-3, doi: 10.1109/OECC.2015.7340235. 
[10] A. Ferrari et al., "The GNPy Open Source Library of Applications for Software Abstraction of WDM Data Transport in Open Optical Networks," 2020 6th IEEE Conference on Network Softwarization (NetSoft), 2020, pp. 386-394, doi: 10.1109/NetSoft48620.2020.9165313.

[11] A. Ferrari et al., "GNPy: an open source planning tool for open optical networks," 2020 International Conference on Optical Network Design and Modeling (ONDM), 2020, pp. 1-6, doi: 10.23919/ONDM48393.2020.9133027. 